\title{
Update in diagnosis and management of primary aldosteronism
}

https://doi.org/10.1515/cclm-2017-0217

Received March 11, 2017; accepted July 24, 2017; previously published online August 28, 2017

Abstract: Primary aldosteronism (PA) is a group of disorders in which aldosterone is excessively produced. These disorders can lead to hypertension, hypokalemia, hypervolemia and metabolic alkalosis. The prevalence of PA ranges from $5 \%$ to $12 \%$ around the globe, and the most common causes are adrenal adenoma and adrenal hyperplasia. The importance of PA recognition arises from the fact that it can have a remarkably adverse cardiovascular and renal impact, which can even result in death. The aldosterone-to-renin ratio (ARR) is the election test for screening PA, and one of the confirmatory tests, such as oral sodium loading (OSL) or saline infusion test (SIT), is in general necessary to confirm the diagnosis. The distinction between adrenal hyperplasia (AH) or aldosteroneproducing adenoma (APA) is essential to select the appropriate treatment. Therefore, in order to identify the subtype of PA, imaging exams such as computed tomography or magnetic ressonance imaging, and/or invasive investigation such as adrenal catheterization must be performed. According to the subtype of PA, optimal treatment - surgical for APA or pharmacological for AH, with drugs like spironolactone and amiloride - must be offered.

Keywords: adrenal vein sampling; arterial hypertension; hypokalemia; oral sodium loading; primary aldosteronism.

\footnotetext{
*Corresponding author: Sandra P. Silveiro, Serviço de Endocrinologia, Hospital de Clínicas de Porto Alegre (HCPA), Rua Ramiro Barcelos, 2350 - Prédio 12, $4^{\circ}$ andar, Porto Alegre, RS 90035-903, Brazil, Phone: +55.51 .33598127 ,

E-mail: ssilveiro@hcpa.edu.br; and Graduate Program in Medical Sciences: Endocrinology, Universidade Federal do Rio Grande do Sul (UFRGS), Porto Alegre, RS, Brazil

Sofia M. Dick, Marina Queiroz, Bárbara L. Bernardi, Angélica Dall'Agnol and Letícia A. Brondani: Graduate Program in Medical Sciences: Endocrinology, Universidade Federal do Rio Grande do Sul (UFRGS), Porto Alegre, RS, Brazil
}

\section{Introduction}

Primary aldosteronism (PA) is a group of disorders in which aldosterone production is inappropriately high for sodium status, is relatively autonomous of the major regulators of secretion (angiotensin II, plasma potassium concentration), and is non-suppressible by sodium loading. Such inappropriate production of aldosterone causes hypertension, cardiovascular damage, sodium retention, suppression of plasma renin, and increased potassium excretion, which may lead to hypokalemia [1].

The first cases of this abnormality were reported by the Polish internist Michał Lityński in 1953, a forgotten author of the first description of primary hyperaldosteronism [2]. Two years later, in 1955, Dr. Jerome W. Conn, a professor of medicine at the University of Michigan, described the syndrome characterized by hypertension, suppressed plasma renin activity (PRA), and increased aldosterone excretion: the syndrome of PA [3]. By 1964, Conn had collected 145 cases, suggesting that up to $20 \%$ of patients with essential hypertension could have PA. Others downplayed this percentage as a gross overestimate. Later, Conn adjusted his predicted prevalence of PA to $10 \%$ of hypertensives, a prediction that was substantiated nearly 40 years later [3].

The prevalence of PA varies according to the population investigated. An Australian retrospective study with 199 subjects with hypertension and normokalemia, provided a minimum incidence for PA of $8.5 \%$, a probable incidence of $12 \%$ and a maximum incidence of $13 \%$ [4]. A retrospective study with a predominantly Chinese hypertensive population, performed in a primary care setting, identified that PA was the underlying cause of hypertension in 5\% of the patients [5]. The PA prevalence in hypertensives (PAPY) study, which recruited 1125 hypertensive subjects from Italian centers, identified a prevalence of $11.2 \%$ of PA [6]. Douma et al., in a retrospective Greek study with patients with resistant hypertension, confirmed PA in $11.3 \%$ of that population. The authors conclude that, since PA is less prevalent in milder forms of hypertension, it is probably less common in patients with 
hypertension than currently thought. PA would therefore be a tertiary endemic, at specialized referral centers in tertiary hospitals, rather than a primary epidemic disorder [7]. To assess the prevalence of PA in primary care practice, a recent study [8] recruited 1672 patients directly from 19 general practitioners in Italy. This study showed a prevalence of $5.9 \%$ of PA, and the overall prevalence of PA increased with the severity of hypertension, from 3.9\% in stage 1 hypertension to $11.8 \%$ in stage 3 hypertension. Finally, a systematic review and meta-regression analysis [9], whose aim was establish the prevalence of PA in hypertensive patients, found that the prevalence estimates varied from $3.2 \%$ to $12.7 \%$ in primary care and from $1 \%$ to $29.8 \%$ in referral centers. Meta-regression analysis showed higher prevalence rates in studies published after 2000, that aimed at assessing prevalence of secondary hypertension, that were retrospective, and that selected consecutive patients, not using a screening test.

The increasing importance of PA recognition arises from the fact that it can have a remarkably adverse cardiovascular and renal impact. It has been clearly demonstrated that PA increases left ventricle telediastolic volume, occasionally associated with increased wall thickness, as compared with similar patients with essential hypertension [10]. In a cross-sectional study that compared the prevalence of cardiovascular events between patients with PA and matched essential hypertension, the odds ratio at PA diagnosis was 2.6 for myocardial infarction, 1.9 for symptomatic re-vascularized ischemic heart disease, 2.9 for symptomatic heart failure, and 5.0 for atrial fibrillation [11]. According to a meta-analysis, the renal impact was expressed by a relative glomerular hyperfiltration at diagnosis of PA, as compared to essential hypertension [12]. Additionally, microalbuminuria was twice more frequent in PA than in essential hypertension at the same blood pressure level [13]. These findings have been attributed to sodium and water retention, inflammation, endothelial dysfunction, cardiac, vascular or renal fibrosis, sympathetic activation, and insulin resistance [10].

Since the appropriate treatment of PA - surgery for adrenal adenoma and carcinoma vs. clinical treatment for adrenal hyperplasia - is highly dependent on the precise diagnosis, it is of utmost importance to accurately state the mechanism of PA. Therefore, the aim of the present review was to update the principles of PA investigation and management, highlighting the screening, confirmatory and localization diagnostic tests, and describing the procedures and concerns regarding each test. Literature search on Pubmed database was performed using the terms: "etiology", "epidemiology", "screening”, "confirmatory tests", "investigation", "management" and "treatment" combined with the words "primary hyperaldosteronism" or "primary aldosteronism".

\section{Pathogenesis}

The two main causes of PA are bilateral adrenal hyperplasia (AH), which represents $60 \%$ of the cases [14], and aldosterone-producing adenomas (APA), contributing to $30 \%$ of the cases [15]. Bilateral adrenal hyperplasia (AH or idiopathic aldosteronism) generally occurs as micronodular (rarely macronodular) hyperplasia. APA, also known as Conn's adenoma, is barely more frequent in female than in male patients. Patients with this form of disease are usually younger than those with bilateral $\mathrm{AH}$ and are more often affected by severe hypertension combined with marked hypokalemia. APAs are frequently unilateral, solitary tumors of $<1.5 \mathrm{~cm}$ in diameter [14].

The uncommon causes of PA include unilateral hyperplasia, aldosterone-producing carcinoma and hereditary forms, such as familial hyperaldosteronism (FH). $\mathrm{FH}$ is an uncommon subtype of PA that has three forms of presentation: FH type I or glucocorticoid-remediable aldosteronism (GRA), FH type II, and FH type III. In a study of 300 consecutive patients with PA, two patients were diagnosed with FH type I (prevalence of 0.66\%). In the remaining 199 families, 12 were diagnosed with FH type II (6\%) [16].

\section{Clinical and laboratory features}

The usual consequences of PA are hypertension, hypokalemia and metabolic alkalosis. The excessive aldosterone secretion leads to an increase in plasma volume and in peripheral vascular resistance, resulting in resistant hypertension [17].

Milliez et al. [18] demonstrated a markedly increased incidence of stroke ( $12.9 \%$ vs. $3.4 \%$ ), non-fatal myocardial infarction ( $4.0 \%$ vs. $0.6 \%)$, and atrial fibrillation $(7.3 \%$ vs. $0.6 \%$ ) in patients with PA compared to those with essential hypertension. About 10 years ago, the association of atrial fibrillation (AF) with hyperaldosteronism was recognized [19].

Only $9 \%-37 \%$ of the patients with PA present hypokalemia, which is due to a renal potassium wasting effect $[17,20,21]$. In most cases, serum potassium is normal and the likelihood of hypokalemia is higher in severe cases [1]. Absence of hypokalemia does not indicate that the patient does not have PA, because reduced serum potassium has low sensitivity for the diagnosis of this disease [1]. In severe cases, usually when serum potassium levels 
are below $2.0 \mathrm{mEq} / \mathrm{L}$, rhabdomyolysis can occur [22]. This feature is a rare manifestation of PA that causes the destruction of a high quantity of striated muscle, leading to changes in the balance of electrolytes and fluids, affecting renal function [22]. In addition, during hypokalemia, nephrogenic diabetes insipidus can occur due to renal tubule antidiuretic hormone resistance, causing mild polyuria, polydipsia and nocturia [17].

A high rate of sodium reabsorption in PA results in hypervolemia. However, peripheral edema is rarely detected if renal and cardiac functions are normal, because after excessive salt retention, the heart releases atrial natriuretic peptide, reducing renal sodium retention [17].

The urinary hydrogen secretion in renal distal tubule produces metabolic alkalosis, which is usually mild, causing no serious consequences, not being noticeable in some cases. Furthermore, mild hypernatremia and hypomagnesemia, probably due to the preservation of proper urinary dilution and concentration abilities of the kidney, can be seen in some patients [17]. Neuromuscular symptoms caused by electrolyte disturbances, such as weakness and paresthesia, rarely occur in these patients [17].

\section{Who should undergo screening for PA?}

The recently published Endocrine Society guidelines recommend the screening of PA in patients who maintain blood pressure (BP) above 150/100 $\mathrm{mmHg}$ after three measurements, performed on different days. In addition, these patients must present refractory hypertension to three standard antihypertensive medications or controlled on four or more antihypertensive drugs. Patients with hypertension associated with spontaneous or diureticinduced hypokalemia, sleep apnea and family history of early onset hypertension or stroke at a young age should be evaluated, as well as all the hypertensive first-degree relatives of patients with PA [1].

It is important to track PA in patients with adrenal incidentalomas, establishing whether the adrenal mass is benign or malignant with magnetic resonance imaging (MRI), computed tomography (CT) or positron emission tomography with 18F-2-deoxy-d-glucose (FDGPET). Adrenal incidentaloma is an adrenal mass detected on imaging studies performed to evaluate symptoms of a condition not related to the adrenal gland. It is wise to evaluate without delay adrenal masses in children, adolescents, pregnant women and adults $<40$ years of age, because of the high probability of malignancy [23].

\section{Case recognition}

The aldosterone-to-renin ratio (ARR), obtained through plasma aldosterone concentration (PAC) and plasma renin activity assay (PRA) or plasma renin concentration (PRC), is the cornerstone for screening PA, being considered the most reliable method for the traceability of the disease [1, 24-29]. The ARR is calculated by dividing the PAC (in $\mathrm{ng} / \mathrm{dL}$ or $\mathrm{pmol} / \mathrm{L}$ ) by the PRA (in $\mathrm{ng} / \mathrm{mL} / \mathrm{h}$ ) or PRC (in $\mathrm{mU} / \mathrm{L}$ or $\mathrm{ng} / \mathrm{L}$ ). Due to the difficulty in calculating the AAR, given that laboratories provide plasma aldosterone in different units of measurement, and renin is measured as PRA or PRC, an app that assists practicing physicians calculating the AAR has been developed and can can be downloaded from the ESH website and the Apple store [30]. The cut-off value employed to define the presence of PA depends on the method used to measure the hormones (Table 1) [31]. ARR has the highest sensitivity and specificity for disease detection and presents advantages in comparison to the isolated PAC measurement, considering pre-analytical factors, such as body posture prior to the collection, diuretics administration and salt intake, besides presenting less intra- and intertest variations [29, 32].

There are many compounds that may interfere in the accuracy of aldosterone measurement $[29,33]$. In the renin-angiotensin-aldosterone system (RAAS), aldosterone is considered a powerful molecule, promoting water and sodium retention, as well as potassium and hydrogen loss [34]. In urine, aldosterone is abundantly presented as aldosterone-18-glucuronide and $3 \alpha-5 \beta$ tetrahydroaldosterone. Therefore, it requires a 24 -h urine sample and acid hydrolysis, or enzymatic methods, for conversion of glucuronide to aldosterone [29].

Radioimmunoassay (RIA) used to be the most employed method to measure aldosterone, but it has progressively been replaced by chemiluminescence (CLIA). An excellent agreement has been demonstrated between CLIA and RIA. In addition, CLIA is a simpler test, with lower costs, and is environment friendly due to the lack

Table 1: ARR cutoff values. ${ }^{a}$

\begin{tabular}{lrrrr}
\hline & $\begin{array}{r}\text { PRA, } \\
\text { ng/mL/h }\end{array}$ & $\begin{array}{r}\text { PRA, } \\
\text { pmol/L/min }\end{array}$ & $\begin{array}{r}\text { PRC, } \\
\text { mU/L }\end{array}$ & $\begin{array}{r}\text { PRC, } \\
\text { ng/L }\end{array}$ \\
\hline PAC, ng/dL & 20 & 1.6 & 2.4 & 3.8 \\
PAC, pmol/L & 750 & 60 & 91 & 144 \\
\hline
\end{tabular}

PAC, plasma aldosterone concentration; PRA, plasma renin activity;

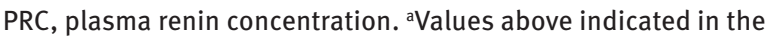
Table suggest the presence of primary aldosteronism. 
of use of radioactivity. More recently, the purification of aldosterone by liquid chromatography-mass spectrometry has allowed an even more consistent measurement of the substance, although not fully accessible. A reference material is not yet available for quality control of aldosterone measurement $[29,35,36]$.

RIA was traditionally used to estimate renin activity, known as PRA [37]. It is a test with high analytical sensitivity, being more precise for smaller renin concentrations. However, long incubation time and consequently long turnaround times are affected by endogenous substrate (angiotensinogen) concentration that may be elevated in certain conditions such as pregnancy [37]. On the other hand, PRC, measured by CLIA, has received increasing attention. Manolopoulou et al. [38] validated the CLIA assay in PA patients, showing that this method is a convenient alternative for the measurement of PAC and PRC on a single automated analyzer. Therefore, PRA evaluated by RIA has been progressively replaced by PRC measured by CLIA in several laboratories, despite lack of any proven superiority of the former over the latter $[26,28,39]$. Encouragingly, a recent Italian study concluded that the area under the curve for ARR based on CLIA was higher than that for RIA [39]. In the same study, the Youden index for ARR based on CLIA (cutoff point) was 2.06, which corresponded to a sensitivity of $92 \%$ and a specificity of $91.6 \%$, and, for the ARR based on RIA, the optimal cutoff value was 38.7 , corresponding to a sensitivity of $80 \%$ and a specificity of $92 \%$ [39]. Hence, CLIA method clearly presents advantages, such as independence on renin substrate availability, handling of plasma at room temperature, more reproducible results, lack of use of radioactivity and the possibility of performing the assay with automation, saving time, costs and human resources [39].

The ARR test may display false-positive or falsenegative results due to multiple interfering factors. If there is any doubt in the interpretation or regarding the intake of drugs, the test should be repeated. A range of pharmacological substances, such as $\beta$-blockers, angiotensin-converting enzyme inhibitors, selective serotonin inhibitors and oral contraceptives influence the screening of the disease (Table 2) [1, 41]. Whenever possible, antihypertensive drugs should be replaced by a longacting calcium-channel blocker (CCB) and/or doxazosin for at least 3 weeks and 6 week for spironolactone.

The body posture is another factor that interferes with ARR test, and various protocols are used: reclined posture, sitting position and erect. Endocrine Society guidelines Highlight that ARR is more sensitive when samples are collected in the morning, after being out of bed for at least $2 \mathrm{~h}$, and after being seated for 5-15 min [1]. Lonati et al. [26]
Table 2: Medications that may lead to false-positive or false-negative ARR results.

\begin{tabular}{|c|c|c|c|}
\hline Medications & $\begin{array}{r}\text { Effect on } \\
\text { plasma } \\
\text { aldosterone }\end{array}$ & $\begin{array}{r}\text { Effect on } \\
\text { plasma } \\
\text { renin }\end{array}$ & $\begin{array}{l}\text { Effect } \\
\text { on ARR }\end{array}$ \\
\hline$\beta$-Blockers & $\downarrow$ & $\downarrow \downarrow$ & $\uparrow(\mathrm{FP})$ \\
\hline $\begin{array}{l}\text { Central agonists } \\
\text { (clonidine, methyldopa) }\end{array}$ & $\downarrow$ & $\downarrow \downarrow$ & $\uparrow(\mathrm{FP})$ \\
\hline NSAIDs & $\downarrow$ & $\downarrow \downarrow$ & $\uparrow(\mathrm{FP})$ \\
\hline $\mathrm{K}^{+}$-Wasting diuretics & $\rightarrow \uparrow$ & $\uparrow \uparrow$ & $\downarrow(\mathrm{FN})$ \\
\hline $\mathrm{K}^{+}$-Sparing diuretics & $\uparrow$ & $\uparrow \uparrow$ & $\downarrow(\mathrm{FN})$ \\
\hline ACE inhibitors & $\downarrow$ & $\uparrow \uparrow$ & $\downarrow(\mathrm{FN})$ \\
\hline ARBs & $\downarrow$ & $\uparrow \uparrow$ & $\downarrow(\mathrm{FN})$ \\
\hline $\mathrm{Ca}^{2+}$ blockers (DHPs) & $\rightarrow \downarrow$ & $\uparrow$ & $\downarrow(\mathrm{FN})$ \\
\hline Renin inhibitors ${ }^{\mathrm{a}}$ & $\downarrow$ & $\downarrow \uparrow$ & $\begin{array}{l}\uparrow(\mathrm{FP}) \\
\downarrow(\mathrm{FN})\end{array}$ \\
\hline Oral contraceptives ${ }^{\mathrm{b}}$ & $\uparrow$ & $\downarrow$ & $\uparrow(\mathrm{FP})$ \\
\hline
\end{tabular}

NSAIDs, nonsteroidal anti-inflammatory drugs; $\mathrm{K}^{+}$, potassium; $A C E$, angiotensin-converting enzyme; ARBs, angiotensin II receptor blockers; DHPs, dihydropyridines; FP, false positive; FN, false negative. aRenin inhibitors lower PRA, but raise PRC. ${ }^{b}$ Combined oral contraceptive increases ARR only if PRC is used to calculate the ratio [40]. (Adapted from Ref. [1].)

compared ARR in a supine position with ARR in an erect position in subjects with arterial hypertension. A significant increase in ARR value was found in erect position for $1 \mathrm{~h}$ when compared to a supine position. However, another study compared the ARR in patients with PA and in patients with arterial hypertension in a supine position at night and erect posture for 1,2 and $4 \mathrm{~h}$. The authors considered that the erect posture for $1 \mathrm{~h}$ was the ideal position, with higher sensitivity and specificity [42]. On the other hand, Barigou et al. [43] showed a strong correlation between ARR in sitting position and ARR in a supine position and erect, indicating that the ARR sitting position could replace the ARR in a supine position in clinical practice.

\section{Exclusion tests}

The ARR, used as a screening test for PA, may not be sufficient to establish the PA diagnosis, especially in terms of specificity [44]. In this context, confirmatory tests are actually used to exclude false-positive results in the screening test, since all available tests present negative predictive values higher than their positive predictive values, considering the prevalence of PA among the selected population. Therefore, this approach is useful to discard, with a significant level of certainty, the disease in those patients who have a negative result, avoiding 
unnecessary costs in imaging and invasive investigation, such as adrenal venous sampling (AVS) [44].

The general recommendation is that the patient should undergo one or more of the following tests, such as oral sodium loading (OSL), saline infusion (SIT), captopril challenge (CCT) or fludrocortisone suppression test (FST) (Table 3) $[1,44]$. However, for patients that present spontaneous hypokalemia, plasma renin below detection levels and $\mathrm{PAC}>20 \mathrm{ng} / \mathrm{dL}(0.555 \mathrm{nmol} / \mathrm{L})$, confirmatory tests are not necessary $[1,44]$.

In fact, based on the current literature, no available test is recognized as the gold standard, since there is no international standardized protocol properly validated for PA confirmation $[1,44,46]$. As pointed out by the French consensus, this scenario may be explained by the following: (a) available studies have small series of patients, (b) there is no validated reference test, (c) lack of consensual cutoffs values for positivity, (d) lack of studies comparing the different types of tests, (e) difficulty in performing saline loading in some clinical conditions and (f) the possibility of false-negative results in cases of aldosterone suppression along the presence of lateralization in AVS [44]. Moreover, the mechanisms of CCT, FST, SIT and OSL are based on the premise that the increased aldosterone secretion in PA patients is autonomous and non-suppressible [44, 47]. However, several studies have already demonstrated that this fallacy may lead to a misdiagnosis. Many APA express type 1 angiotensin II receptors, and for that reason, can be responsive to changes in renin-angiotensin system. In this context, many potentially curable patients may not be considered for surgical treatment based on false-negative results [47-49]. Therefore, in particular cases of patients seeking surgical cure and with high ARR, proceeding directly to imaging and AVS should be considered, or repeating the screening ARR under strict protocol conditions in those whose first ARR was inconclusive [47].

Although some studies have suggested differences among these tests in terms of sensitivity, specificity and reliability, there is no definitive evidence to recommend one over the other, and the choice is usually based on patient compliance, cost, laboratory routine and local expertise [1].

\section{Subtype classification}

The hypersecretion of aldosterone in PA can be either bilateral, in the case of adrenal hyperplasia (AH), or unilateral, usually due to aldosterone-producing adenoma 
(APA) and unilateral adrenal hyperplasia [50, 51]. The distinction between these presentations is essential to select the appropriate treatment, which can be surgical and potentially lead to the cure in unilateral PA, or to a lifelong pharmacological treatment in the case of bilateral disease $[1,50,52]$. In order to identify the subtype of PA, computed tomography (CT)/magnetic resonance imaging (MRI) and adrenal vein sampling (AVS) should be performed.

\section{Adrenal imaging}

Adrenal imaging can be useful before proceeding to further invasive subtype investigation. CT is mainly recommended to exclude large masses (e.g. $>4 \mathrm{~cm}$ ) that may represent aldosterone-producing carcinoma and to provide anatomical information, such as the localization of the right adrenal vein for posterior catheterization [1]. However, this method has several limitations. Hyperplastic adrenal glands are usually normal on CT, and micro-aldosterone-producing adenomas are not always detectable $[1,51]$ or may be considered to be part of bilateral nodular hyperplasia. Therefore, misdiagnosis on both directions is not uncommon. Furthermore, CT findings cannot provide information about the secretory activity of nodules - that may be nonfunctional, especially in patients who are older than 35 years [1, 50-52] - which increases the likelihood of false-positive results.

A systematic review of 38 studies, including 950 patients, showed that in $37.8 \%$ of cases, CT/MRI results did not agree with AVS results, leading to inappropriate adrenalectomy in $14.6 \%$, inappropriate medical treatment in $19.1 \%$ and adrenalectomy in the wrong side in $3.9 \%$ [53]. Furthermore, it has been demonstrated that in patients who underwent unilateral adrenalectomy, imaging were concordant to the surgically documented side in $58.6 \%$, while AVS was in 97.1\% [54]. Similar results were found in a retrospective study conducted by Ladurner and coworkers that showed preoperative MRI or CT imaging are insufficient for a correct lateralization of primary aldosteronism. Thus, AVS represents an essential diagnostic step to distinguish between unilateral and bilateral disease [55].

MRI is reserved for cases in which CT is not indicated, as it is more expensive and has inferior spatial resolution than CT [52].

\section{Adrenal vein sampling (AVS)}

Adrenal venous sampling (AVS) is the most reliable method to identify the subtype of PA, with $95 \%$ and
$100 \%$ of sensitivity and specificity, respectively. Therefore, the last Endocrine Society guideline recommends proceeding with AVS to implement the most adequate treatment in those patients who seek a potential surgical cure for PA [1].

Despite efforts to assess the cause of PA with noninvasive methods, such as SIT, they are in fact unable to discriminate between APA and AH [56]. It has been suggested that in younger patients with marked PA (spontaneous hypokalemia, plasma aldosterone concentration $>30 \mathrm{ng} / \mathrm{dL}$ [0.831 nmol/L]) and CT findings of probable solitary unilateral adenoma, the AVS would not be necessary to indicate the adrenalectomy $[1,54]$ (Figure 1). However, this is quite a weak suggestion, since it was based in the results of a small retrospective observational study. Familial PA types I and III in young patients with PA or suggestive familiar history should be considered and excluded before proceeding to the AVS [51].

Although there is enough evidence to support the use of AVS, this is still an underused procedure due to the technical difficulties, to the potential risks involved such as adrenal vein rupture $(0.6 \%)$, and due to the lack of accepted criteria to interpret its results [51, 52, 57]. Moreover, there are no uniform standards to perform AVS procedures among different centers $[1,51]$. The variability of the techniques includes (a) simultaneous or sequential bilateral catheterization without stimulation; (b) unstimulated sequential or simultaneous bilateral catheterization followed by bolus cosyntropin-stimulated sequential or

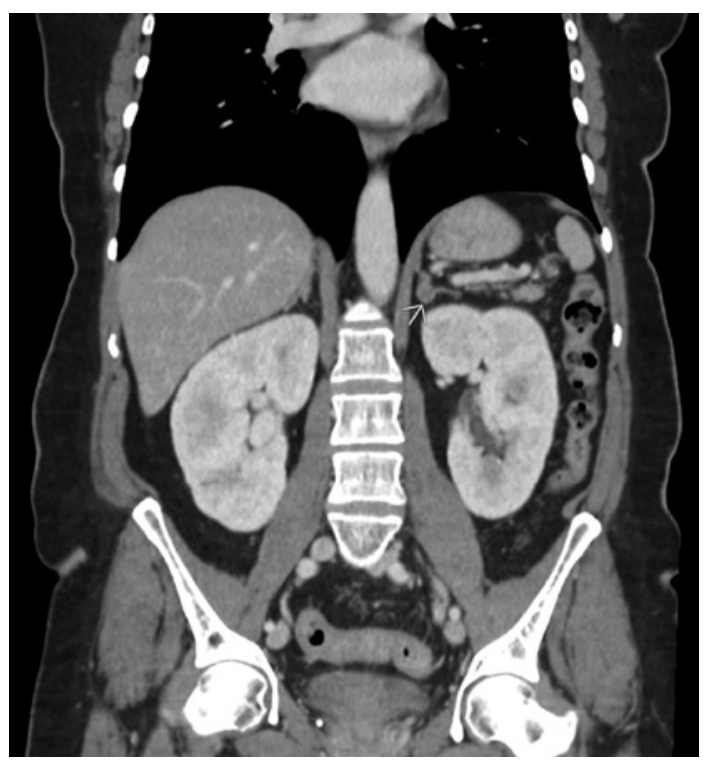

Figure 1: CT image in a coronal view of left adrenal gland, showing an adenoma (arrow) with approximately $1 \mathrm{~cm}$ (with permission of the patient). 
simultaneous bilateral AVS ( $250 \mu \mathrm{g}$ of cosyntropin); or (c) continuous cosyntropin stimulation (infusion of $50 \mu \mathrm{g} / \mathrm{h}$, $30 \mathrm{~min}$ before catheterization and during the procedure) with sequential bilateral AVS [1].

Continuous cosyntropin stimulation is recommended by some groups in order to reduce fluctuations in aldosterone secretion caused by stress during sequential catheterization, to maximize the selectivity index to confirm successful sampling of adrenal vein and finally to maximize aldosterone secretion from an APA during the AVS [1,51]. However, although cosyntropin use increases the selectivity index, there are evidence in literature demonstrating that this approach leads to inconsistent results due to variable responses of adrenal gland to cosyntropin, whether or not APA is present [58], and to the lack of conclusive evidence about its superiority to determine lateralization of aldosterone excess [51]. Moreover, the use of cosyntropin could stimulate aldosterone secretion of the contralateral adrenal from an APA as well, masking a unilateral type of PA [59]. Therefore, it has been demonstrated that the systematic use of cosyntropin stimulation does not improve the diagnostic accuracy of AVS.

When cosyntropin infusion is not employed, the AVS should be performed in the morning, after being in supine position overnight, in order to avoid changes in aldosterone levels that may occur in patients with angiotensin II-responsive varieties of PA and to take advantage of endogenous corticotropin levels on aldosterone levels in all subtypes of PA [1].

Some centers use simultaneous AVS technique to avoid the fluctuation differences in aldosterone concentration $[1,51,57]$. In fact, the simultaneous catheterization of adrenal veins was proven to provide more reliable results, since more than a half of the patients undergoing AVS present an ACTH-induced stress response, causing an increased cortisol release, which may lead to misinterpretation of the selectivity index when sequential catheterization is used [60].

Before the procedure, medications should be withdrawn for at least 3 weeks; 6 weeks for mineralocorticoid receptor (MR) antagonists [52], which may lead to a stimulation of the opposite adrenal gland of an APA, masking the unilateral aldosterone production [1]. Plasma renin or renin activity can be measured in order to verify the hormone suppression, and then proceed to the AVS independently when the drug has been withdrawn [52]. It is also important to correct hypokalemia with oral or intravenous potassium before AVS, since hypokalemia may lead to a decrease in aldosterone secretion and potentially mask a unilateral APA [53].
The adrenal vein catheterization procedure is usually performed by an interventional radiologist, using percutaneous femoral vein approach. The position of the catheter is verified using non-ionic contrasted radiography, and the selectivity index through cortisol concentrations from adrenal veins and peripheral veins (Figure 2). Significant improvements in the diagnostic performance of AVS were possible after the introduction of selectivity index and lateralization index, which allowed the confirmation of the correct catheterized site and the detection of lateralized aldosterone excess when present, respectively [61]. However, data in literature regarding the best cut-off values of the indices for the accurate identification of the successfully executed procedures as well as the identification of potentially curable patients with APA remain controversial [62]. Table 4 simplifies the interpretation of AVS results.

(i) Selectivity index (SI): Used to assess the selectivity of adrenal vein catheterization - the ratio between the concentration of plasma cortisol in the adrenal vein and in the inferior vena cava [50,51,57]. The suggested cutoff value for the SI obtained under unstimulated condition is $\geq 2$, and when performed with cosyntropin stimulation, $\geq 3$ [51].

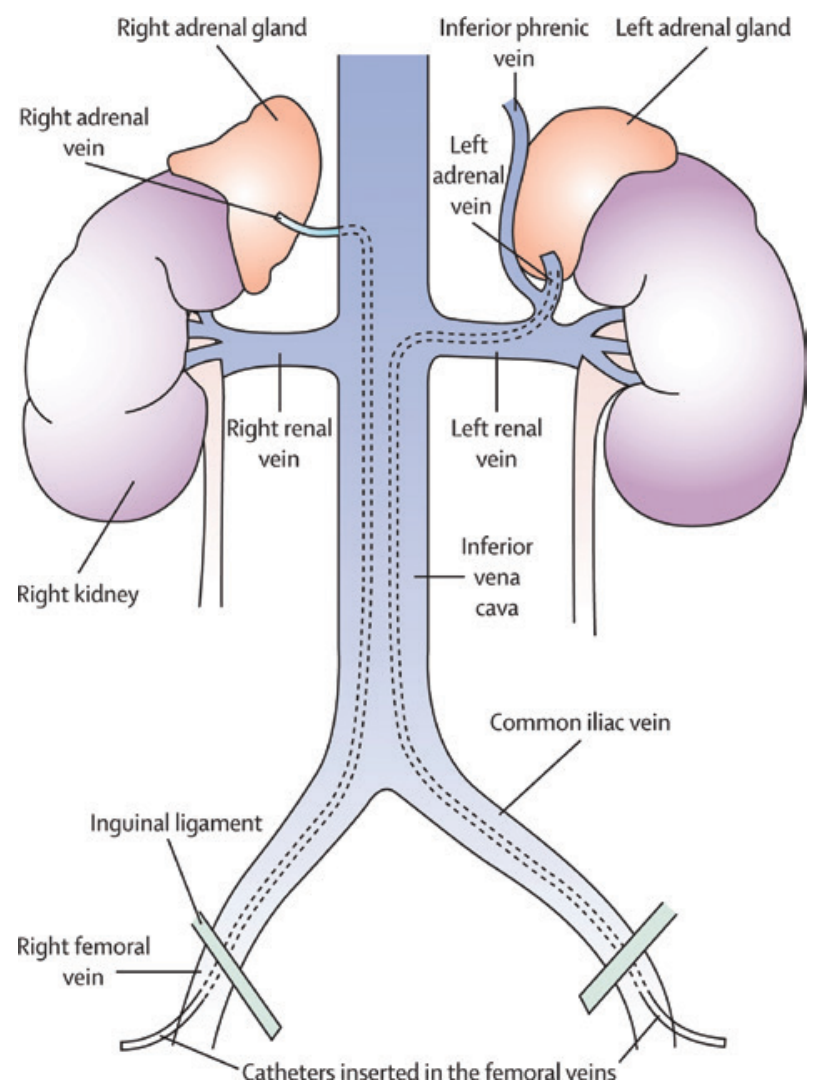

Figure 2: Schematic description of adrenal vein anatomy and adrenal vein sampling [52]. 
Table 4: Interpretation of AVS results.

\author{
Selectivity index: \\ PCC each side/PCCIVC \\ Lateralization index: \\ PAC dom side/PCC dom side:PAC nond side/ \\ PCC nond side \\ Contralateral suppression index: \\ PAC nond side/PCC nond side:PACIVC/PCCIVC
}

Relative aldosterone secretion index:

PAC side/PACIVC : PCC side/PCCIVC

\author{
$>2$ or $>3$ (in use of ACTH) confirms the correct catheterization of adrenal vein \\ $>2$ or $>4$ (in use of ACTH) demonstrates lateralized aldosterone excess and \\ $<3$ indicate bilateral PA \\ $<1$ indicates aldosterone suppression in non-dominant side, which is \\ consistent to aldosterone overproduction in the other side
}

$<0.9$ considering non-dominant side excludes non-APA cases or ipsilateral APA

PCC, plasma cortisol concentration; PAC plasma aldosterone concentration; IVC, inferior vena cava; side: referred to the adrenal vein; nondominant side (nond): side with lower PAC; dominant side (dom): side with higher PAC [44, 51, 58].

(ii) Lateralization index (LI): Used to establish whether a lateralized aldosterone excess exists - aldosterone/cortisol on the dominant side: aldosterone/ cortisol on the non-dominant side [50,51,57]. The aldosterone plasma concentrations from each side are divided by their respective cortisol concentrations in order to avoid potential dilution effects. If the procedure is performed with cosyntropin stimulation, the lateralization is confirmed if the cutoff of cortisol-corrected aldosterone ratio from dominant to non-dominant side is more than 4:1 [1, 63]; bilateral aldosterone hypersecretion is probable considering a ratio of $<3: 1[1,52]$. The ratios between 3:1 and 4:1 must be interpreted carefully in the context of clinical presentation, CT findings, ancillary tests and another AVS should be executed if it is possible [1]. There is evidence demonstrating that values of LI between 2 and 4 may be present in patients without confirmed PA [63]. Without the cosyntropin stimulation, the lateralization cutoff to determine unilateral disease is considered to be more than 2:1 by some centers. Other groups consider the lateralization confirmed when the aldosterone-cortisol ratio in the dominant adrenal vein is at least 2.5 times greater than the aldosterone-cortisol simultaneously collected in the peripheral vein and the contralateral adrenal vein aldosterone-cortisol ratio is no higher than peripheral [1].

(iii) Contralateral suppression index (CSI) : Used to determine the aldosterone suppression in non-dominant adrenal side that suggests aldosterone overproduction in the opposite side [52]. Contralateral suppression is considered when the aldosterone to cortisol ratio in the non-dominant adrenal is less than the ratio in the inferior vena cava (IVC) or in the peripheral vein sample $(\mathrm{CSI}<1)[52]$. CSI can be used when cannulation of the other adrenal vein is not possible or when LI is between 3:1 and 4:1 [51, 52]. There is evidence demonstrating that the presence of CSI may be a predictor of successful surgical resolution of hypertension in PA patients [64]. However, CSI by itself is not enough to predict unilateral $\mathrm{PA}$, since up to $30 \%$ of patients with bilateral $\mathrm{AH}$ showed suppression with CSI $[52,58]$. In addition, it should be considered that aldosterone hyperproduction from an APA is frequently insufficient to suppress aldosterone production from the contralateral adrenal gland [52].

(iv) Relative aldosterone secretion index (RASI): This novel index is obtained with PAC in the adrenal vein to PAC in the IVC ratio divided by selectivity index in the same side. RASI can be useful in unilaterally selective AVS procedures, as demonstrated in a study [58] that found a specificity of $100 \%$ in excluding non-APA cases or ipsilateral APA, when considering non-dominant side RASI of 0.9 or less after metoclopramide aldosterone stimulation. In addition, the index can help to unmask potential misleading suppression of aldosterone in patients without unilateral PA, once no non-APA patient showed a RASI 0.9 or less on the non-dominant side [58].

\section{Treatment}

For patients with documented unilateral PA, unilateral laparoscopic adrenalectomy is the recommended treatment. If a patient is unable or unwilling to undergo surgery, medical treatment is proposed, including a MR antagonist (Table 5). If an ARR-positive patient is reluctant or unable to undergo further investigation, medical treatment including an MR antagonist is similarly recommended. 


\section{Pharmacological treatment}

\section{Spironolactone}

Spironolactone represents the first-line medical treatment of PA and the literature testifies its efficacy not only with respect to hypertension, but also to protection of several target organs. It is a competitive antagonist of both aldosterone and androgen receptors and behaves as a weak agonist of the progesterone receptor. Spironolactone inhibits sodium reabsorption in the basolateral membrane of the principal cells of the renal collecting duct, directly by inhibiting $\mathrm{Na} / \mathrm{K}$ ATPase and indirectly by inhibiting epithelial sodium channel. The resultant potassium retention makes spironolactone a potassium-sparing diuretic [13]. The natriuretic effect persists for several days after cessation of the drug, while the effects on renin secretion may take several weeks to disappear. Therefore, when patients need biochemical assessment for PA, cessation of spironolactone treatment for at least 6 weeks is recommended [65]. Despite a clearly demonstrated dose-response relation for spironolactone side effects, a significant incidence of adverse effects is found at $25-50 \mathrm{mg} /$ day. One study reported $7 \%$ incidence of gynecomastia at 6 months for doses $<50 \mathrm{mg} /$ day and $52 \%$ incidence for $>150 \mathrm{mg} /$ day [17].

\section{Eplerenone}

Eplerenone a selective MR antagonist without antiandrogen and progesterone agonist effects, thus reducing the rate of adverse endocrine side effects [1]. Eplerenone in vivo has $50 \%$ of the MR antagonist potency of spironolactone. Its better tolerability profile needs to be balanced against its higher cost and the possibility that spironolactone may lower BP more effectively than eplerenone in the medical treatment of PA [1]. Eplerenone is a good alternative in case of side effects of spironolactone [66].

\section{Amiloride}

Amiloride is an epithelial sodium channel blocker (ENacblocker) used as a potassium-sparing diuretic that can ameliorate hypertension and hypokalemia in patients with PA. It is generally well tolerated and lacks the sex steroid-related side effects of spironolactone, but it does not provide the beneficial effects on endothelial function [21, 22]. In mild cases or when the patient does not tolerate spironolactone or eplerenone side effects, ENaC-blockers may be added to MR antagonists in order to reduce the doses and thus the side effects of these drugs [67]. 


\section{Calcium-channel blockers (CCB), angiotensin converting enzyme (ACE) inhibitors and angiotensin receptor blockers (ARB)}

They have been evaluated in very few patients with PA, and in general, they are antihypertensive drugs without a major effect on MR activation. They are, nevertheless, commonly used to lower BP (in combination with MR antagonists) if BP remains above normal [68].

\section{New agents modulating the renin-angiotensin-aldosterone system}

\section{Finerenone}

Although finerenone competitively antagonizes the MR, it shows more natriuretic effects than spironolactone and eplerenone. The safety and tolerability of finerenone was studied during the mineralocorticoid-receptor antagonist tolerability study (ARTS) in patients with heart failure and mild/moderate chronic kidney disease, showing less hyperkalemia when compared with spironolactone. The longterm effects of finerenone will be investigated in a phase III study for the treatment of chronic heart failure [69].

\section{Aldosterone synthase inhibitors (ASIs)}

The key enzyme in aldosterone production is aldosterone synthase (CYP11B2). CYP11B2 is predominantly expressed in the adrenal gland, but it is also expressed in the cardiovascular system and brain. FAD286, the R-enantiomer of fadrozole, was initially used as a drug to treat breast cancer, and showed benefits in therapy of cardiovascular disorders in experimental models of cardiovascular diseases and diabetes. Phase II studies with another agent, LCI699, showed that in patients with primary hyperaldosteronism characterized by severe hypertension and hypokalemia, there was a reversible and dose-dependent $70 \%-80 \%$ decrease in plasma and urinary aldosterone concentrations with a massive accumulation of the aldosterone precursor, deoxycorticosterone, in the plasma [69].

\section{Surgical treatment}

Unilateral laparoscopic adrenalectomy is the preferred management approach for most patients with localized aldosterone-producing adenoma and unilateral adrenal hyperplasia, because this treatment effectively normalizes endogenous aldosterone secretion and hypokalemia, both improving BP and reducing the number of drugs required to control $\mathrm{BP}$, as well as being cost-effective [1, 70]. The benefit of this procedure is much higher than its risks [1]. However, increased BP can persist after adrenalectomy, usually when the primary hypertension coexists with PA and the patient is older and/or presents long-term hypertension [71, 72]. An effective patient cure (BP $<140 / 90 \mathrm{mmHg}$ ) can be negatively affected by numerous factors, such as family history of hypertension, more than 5 years' duration of hypertension, presence of hypokalemia, age $>50$ years, low urinary aldosterone secretion, presence of increased serum creatinine, reduced response to spironolactone and preoperative requirement of two or more antihypertensive medications [54, 73-76].

Prior to surgery, both hypertension and hypokalemia should be well controlled, sometimes requiring a delay in surgery and the addition of an MR antagonist. In the postoperative process, plasma aldosterone and renin activity levels should be shortly measured after surgery, to check if there was a satisfactory biochemical response; nevertheless, notice that renin levels may not reduce immediately. Clinicians should also interrupt potassium supplementation and spironolactone and, if appropriate, reduce antihypertensive therapy on postoperative day 1 . Furthermore, during the first few weeks after surgery, a generous sodium diet is recommended in order to avoid hyperkalemia. This phenomenon is due to the hypoaldosteronism that originated from chronic contralateral adrenal gland suppression. Persistent hypoaldosteronism may occur in up to 5\% of adrenalectomized patients, requiring mineralocorticoid replacement therapy with fludrocortisone [1].

\section{Conclusion and perspectives}

Since the ARR is the cornerstone of PA screening, health professionals should be aware of the increasing worldwide tendency of replacing the evaluation of plasma renin activity by the direct measurement of renin concentration. Such change hugely modifies cutoff points of ARR in the order of 10 times, from about 20 to 2 as the novel cutoff using renin concentration. Regarding the so called "confirmation tests", such as saline infusion test, these are actually employed as an exclusion tool, since they present an elevated negative predictive value, therefore eliminating the possibility of disease in patients with negative results. These tests are required to avoid further invasive investigation in cases of false-positive screening. Concerning imaging, CT is mainly recommended to exclude 
large masses that may represent aldosterone-producing carcinoma, and also to provide vascular anatomical information prior to catheterization. At this point, subsequent adrenal vein sampling (AVS) is usually indicated, in order to identify unilateral or bilateral aldosterone overproduction. The relative aldosterone secretion index (RASI) is a novel index, with a specificity of $100 \%$ that has enhanced the diagnostic performance of the examination. Proper evaluation and timely treatment of primary aldosteronism definitely improves the prognosis of these patients.

Author contributions: All the authors have accepted responsibility for the entire content of this submitted manuscript and approved submission.

Research funding: This work was supported by Hospital de Clínicas de Porto Alegre - Fundo de Incentivo a Pesquisa e Eventos and Graduate Program in Medical Sciences: Endocrinology, Universidade Federal do Rio Grande do Sul (UFRGS), Porto Alegre, RS, Brazil.

Employment or leadership: None declared.

Honorarium: None declared.

Competing interests: The funding organization played no role in the study design; in the collection, analysis, and interpretation of data; in the writing of the report, or in the decision to submit the report for publication.

\section{References}

1. Funder JW, Carey RM, Mantero F, Murad MH, Reincke M, Shibata H, et al. The management of primary aldosteronism: case detection, diagnosis, and treatment: an Endocrine Society Clinical Practice guideline. J Clin Endocrinol Metab 2016;101:1889-916.

2. Kucharz EJ. Michał Lityński, a forgotten author of the first description of primary hyperaldosteronism. Pol Arch Med Wewn 2007;117:57-8.

3. Young WF. Primary aldosteronism: renaissance of a syndrome. Clin Endocrinol 2007;669:607-18.

4. Gordon RD, Stowasser MD, Tunny TJ, Klemm SA, Rutherford JC. High incidence in primary aldosteronism in 199 patients referred with hypertension. Clin Exp Pharmacol Physiol 1994;21:315-8.

5. Loh KC, Koay ES, Khaw MC, Emmanuel SC, Young HF Jr. Prevalence of primary aldosteronism among Asian hypertensive patients in Singapore. J Clin Endocrinol Metab 2000;85:2854-9.

6. Rossi GP, Bernini G, Caliumi C, Desideri G, Fabris B, Ferri C, et al. A prospective study of the prevalence of primary aldosteronism in 1,125 hypertensive patients. J Am Coll Cardiol 2006;48:2293-300.

7. Douma S, Petidis K, Doumas M, Papaefthimiou P, Triantafyllou A, Kartali N, et al. Prevalence of primary hyperaldosteronism in resistant hypertension: a retrospective observational study. Lancet 2008;371:1921-6.
8. Monticone S, Burrello J, Tizzani D, Bertello C, Viola A, Buffolo F, et al. Prevalence and clinical manifestations of primary aldosteronism encountered in primary care practice. J Am Coll Cardiol 2017;69:1811-20.

9. Käyser S, Dekkers T, Groenewoud HJ, van der Wilt GJ, Carel Bakx J, van der Wel MC, et al. Study heterogeneity and estimation of prevalence of primary aldosteronism: a systematic review and meta-regression analysis. J Clin Endocrinol Metab 2016;101:2826-35.

10. Baguet JP, Steichen O, Mounier-Véhier C, Gosse P. SFE/SFHTA/ AFCE consensus on primary aldosteronism, part 1: Epidemiology of PA, who should be screened for sporadic PA? Ann Endocrinol (Paris) 2016;77:187-91.

11. Savard S, Amar L, Plouin PF, Steichen O. Cardiovascular complications associated with primary aldosteronism: a controlled cross-sectional study. Hypertension 2013;62:331-6.

12. Kuo CC, Wu VC, Tsai CW, Wu KD. Relative kidney hyperfiltration in primary aldosteronism: a meta-analysis. J Renin Angiotensin Aldosterone Syst 2011;12:113-22.

13. Rossi GP, Bernini G, Desideri G, Fabris B, Ferri C, Giacchetti G, et al. Renal damage in primary aldosteronism: results of the PAPY Study. Hypertension 2006;48:232-8.

14. Schirpenbach C, Reincke M. Primary aldosteronism: current knowledge and controversies in Conn's syndrome. Nat Clin Pract Endocrinol Metab 2007;3:220-7.

15. Åkerström T, Maharjan R, Willenberg HS, Cupisti K, Ip J, Moser A, et al. Activating mutations in CTNNB1 in aldosterone producing adenomas. Sci Rep 2016;6:19546.

16. Mulatero P, Tizzani D, Viola A, Bertello C, Monticone S, Mengozzi $\mathrm{G}$, et al. Prevalence and characteristics of familial hyperaldosteronism: the PATOGEN study (Primary Aldosteronism in TOrinoGENetic forms). Hypertension 2011;58:797-803.

17. Pechère-Bertschi $A$, Herpin $D$, Lefebvre H. SFE/SFHTA/ AFCE consensus on primary aldosteronism, part 7: medical treatment of primary aldosteronism. Ann Endocrinol (Paris) 2016;77:226-34.

18. Milliez P, Girerd X, Plouin PF, Blacher J, Safar ME, Mourad JJ. Evidence for an increased rate of cardiovascular events in patients with primary aldosteronism. J Am Coll Cardiol 2005;45:1243-8.

19. Seccia TM, Caroccia B, Adler GK, Maiolino G, Cesari M, Rossi GP. Arterial hypertension, atrial fibrillation, and hyperaldosteronism: the triple trouble. Hypertension 2017;69:545-50.

20. Parthasarathy HK, Menard J, White WB, Young WF Jr, Williams $\mathrm{GH}$, Williams B, et al. A double-blind, randomized study comparing the antihypertensive effect of eplerenone and spironolactone in patients with hypertension and evidence of primary aldosteronism. J Hypertens 2011;29:980-90.

21. Farquharson CA, Struthers AD. Spironolactone increases nitric oxide bioactivity, improves endothelial vasodilator dysfunction, and suppresses vascular angiotensin I/angiotensin II conversion in patients with chronic heart failure. Circulation 2000;101:594-7.

22. Farquharson CA, Struthers AD. Increasing plasma potassium with amiloride shortens the QT interval and reduces ventricular extrasystoles but does not change endothelial function or heart rate variability in chronic heart failure. Heart 2002;88:475-80.

23. Sywak M, Pasieka JL. Long-term follow-up and cost benefit of adrenalectomy in patients with primary aldosteronism. Br J Surg 2002;89:1587-93. 
24. Harvey AM. Hyperaldosteronism: diagnosis, lateralization, and treatment. Surg Clin N Am 2014;94:643-56.

25. Kumar B, Swee M. Aldosterone-renin ratio in the assessment of primary aldosteronism. J Am Med Assoc 2014;312:184-5.

26. Lonati C, Bassani N, Gritti A, Biganzoli E, Morganti A. Measurement of plasma renin concentration instead of plasma renin activity decreases the positive aldosterone-to-renin ratio tests in treated patients with essential hypertension. J Hypertens 2014;32:627-34.

27. Stowasser P, Taylor PJ, Pimenta E, Ahmed AH, Gordon RD. Laboratory investigation of primary aldosteronism. Clin Biochem Rev 2010;31:39-56.

28. Juutilainen A, Savolainen K, Romppanen J, Turpeinen U, Hämäläinen E, Kemppainen J, et al. Combination of LC-MS/MS aldosterone and automated direct renin in screening for primary aldosteronism. Clin Chim Acta 2014;433:209-15.

29. Rehan M, Raizman JE, Cavalier E, Don-Wauchope AC, Holmes DT. Laboratory challenges in primary aldosteronism screening and diagnosis. Clin Biochem 2015;48:377-87.

30. Rossi GP, Bisogni V. A useful tool to improve the case detection rate of primary aldosteronism: the aldosterone-renin ratio (ARR)-App. J Hypertens 2016;34:1019-21.

31. Galati SJ. Primary aldosteronism challenges in diagnosis and management. Endocrinol Metab Clin N Am 2015;44:355-69.

32. Ferrari P, Shaw SG, Nicod J, Saner E, Nussberger J. Active renin versus plasma renin activity to define aldosterone-to-renin ratio for primary aldosteronism. J Hypertens 2004;22:377-81.

33. Muñoz-Durango N, Fuentes CA, Castillo AE, González-Gómez LM, Vecchiola A, Fardella CE, et al. Role of the renin-angiotensin-aldosterone system beyond blood pressure regulation: molecular and cellular mechanisms involved in end-organ damage during arterial hypertension. Int J Mol Sci 2016;17:797.

34. Spät A, Hunyady L. Control of aldosterone secretion: a model for convergence in cellular signaling pathways. Physiol Rev 2004;84:489-539.

35. Stabler TV, Siegel AL. Chemiluminescence immunoassay of aldosterone in serum. Clin Chem 1991;37:1987-9.

36. Mayes D, Furuyama S, Kem DC, Nugent CA. A radioimmunoassay for plasma aldosterone. J Clin Endocrinol Metab 1970;30:682-5.

37. Wedatilake YN, Scanlon MJ, Barnes SC. The clinical utility of two renin mass methods to detect primary hyperaldosteronism compared with renin activity. Ann Clin Biochem 2011;48:256-62.

38. Manolopoulou J, Fischer E, Dietz A, Diederich S, Holmes D, Junnila $R$, et al. Clinical validation for the aldosterone-to-renin ratio and aldosterone suppression testing using simultaneous fully automated chemiluminescence immunoassays. J Hypertens 2015;33:2500-11.

39. Rossi GP, Ceolotto G, Rossitto G, Seccia TM, Maiolino G, Berton C, et al. Prospective validation of an automated chemiluminescence-based assay of renin and aldosterone for the work-up of arterial hypertension. Clin Chem Lab Med 2016;54:1441-50.

40. Ahmed AH, Gordon RD, Taylor PJ, Ward G, Pimenta E, Stowasser M. Effect of contraceptives on aldosterone/renin ratio may vary according to the components of contraceptive, renin assay method, and possibly route of administration. J Clin Endocrinol Metab 2011;96:1797-804.
41. Aronova A, Fahey III TJ, Zarnegar R. Management of hypertension in primary aldosteronism. World J Cardiol 2014;6:227-33.

42. Yin G, Zhang S, Yan L, Wu M, Xu M, Li F, et al. One-hour upright posture is an ideal position for serum aldosterone concentration and plasma renin activity measuring on primary aldosteronism screening. Exp Clin Endocrinol Diabetes 2012;120:388-94.

43. Barigou M, Ah-Kang F, Orloff E, Amar J, Chamontin B, Bouhanick B. Effect of postural changes on aldosterone to plasma renin ratio in patients with suspected secondary hypertension. Ann Cardiol Angeiol (Paris) 2015;64:169-74.

44. Reznik Y, Amar L, Tabarin A. SFE/SFHTA/AFCE consensus on primary aldosteronism, part 3: confirmatory testing. Ann Endocrinol (Paris) 2016;77:202-7.

45. Ahmed AH, Cowley D, Wolley M, Gordon RD, Xu S, Taylor PJ, et al. Seated saline suppression testing for the diagnosis of primary aldosteronism: a preliminary study. J Clin Endocrinol Metab 2014;99:2745-53.

46. Nanba K, Tamanaha T, Nakao K, Kawashima ST, Usui T, Tagami T, et al. Confirmatory testing in primary aldosteronism. J Clin Endocrinol Metab 2012;97:1688-94.

47. Rossi GP, Seccia TM, Pessina AC. A diagnostic algorithmthe holy grail of primary aldosteronism. Nat Rev Endocrinol 2011;7:697-9.

48. Gordon RD, Gomez-Sanchez CE, Hamlet SM, Tunny TJ, Klemm $\mathrm{SA}$. Angiotensin-responsive aldosterone-producing adenoma masquerades as idiopathic hyperaldosteronism (IHA: adrenal hyperplasia) or low-renin essential hypertension. J Hypertens Suppl 1987;5:S103-6.

49. Phillips JL, Walther MM, Pezzullo JC, Rayford W, Choyke PL, Berman AA, et al. Predictive value of preoperative tests in discriminating bilateral adrenal hyperplasia from an aldosterone-producing adrenal adenoma. J Clin Endocrinol Metab 2000;85:4526-33.

50. Bardet S, Chamontin B, Douillard C, Pagny JY, Hernigou A, Joffre $F$, et al. SFE/SFHTA/AFCE consensus on primary aldosteronism, part 4: subtype diagnosis. Ann Endocrinol (Paris) 2016;77:208-13.

51. Rossi GP, Auchus RJ, Brown M, Lenders JW, Naruse M, Plouin $P F$, et al. An expert consensus statement on use of adrenal vein sampling for the subtyping of primary aldosteronism. Hypertension 2014;63:151-60.

52. Monticone S, Viola A, Rossato D, Veglio F, Reincke M, Gomez-Sanchez C, et al. Adrenal vein sampling in primary aldosteronism: towards a standardized protocol. Lancet Diabet Endocrinol 2015;3:296-303.

53. Kempers MJ, Lenders JW, van Outheusden L, van der Wilt GJ, Schultze Kool LJ, Hermus AR, et al. Systematic review: diagnostic procedures to differentiate unilateral from bilateral adrenal abnormality in primary aldosteronism. Ann Intern Med 2009;151:329-37.

54. Lim V, Guo Q, Grant CS, Thompson GB, Richards ML, Farley DR, et al. Accuracy of adrenal imaging and adrenal venous sampling in predicting surgical cure of primary aldosteronism. J Clin Endocrinol Metab 2014;99:2712-9.

55. Ladurner R, Sommerey S, Buechner S, Dietz A, Degenhart C, Hallfeldt $\mathrm{K}$, et al. Accuracy of adrenal imaging and adrenal venous sampling in diagnosing unilateral primary aldosteronism. Eur J Clin Invest 2017;47:372-7. 
56. Rossi GP, Belfiore A, Bernini G, Desideri G, Fabris B, Ferri C, et al. Prospective evaluation of the saline infusion test for excluding primary aldosteronism due to aldosterone-producing adenoma. J Hypertens 2007;25:1433-42.

57. Rossi GP, Barisa M, Allolio B, Auchus RJ, Amar L, Cohen D, et al. The adrenal vein sampling international study (AVIS) for identifying the major subtypes of primary aldosteronism. J Clin Endocrinol Metab 2012;97:1606-14.

58. Rossitto G, Miotto D, Battistel M, Barbiero G, Maiolino G, Bisogni $\mathrm{V}$, et al. Metoclopramide unmasks potentially misleading contralateral suppression in patients undergoing adrenal vein sampling for primary aldosteronism. J Hypertens 2016;34:2258-65.

59. Mulatero P, Bertello C, Sukor N, Gordon R, Rossato D, Daunt N, et al. Impact of different diagnostic criteria during adrenal vein sampling on reproducibility of subtype diagnosis in patients with primary aldosteronism. Hypertension 2010;55:667-73.

60. Seccia TM, Miotto D, Battistel M, Motta R, Barisa M, Maniero $C$, et al. A stress reaction affects assessment of selectivity of adrenal venous sampling and of lateralization of aldosterone excess in primary aldosteronism. Eur J Endocrinol 2012;166:869-75.

61. Rossi GP, Sacchetto A, Chiesura-Corona M, De Toni R, Gallina $M$, Feltrin GP, et al. Identification of the etiology of primary aldosteronism with adrenal vein sampling in patients with equivocal computed tomography and magnetic resonance findings: results in 104 consecutive cases. J Clin Endocrinol Metab 2001;86:1083-90.

62. Rossi GP, Pitter G, Bernante P, Motta R, Feltrin G, Miotto D. Adrenal vein sampling for primary aldosteronism: the assessment of selectivity and lateralization of aldosterone excess baseline and after adrenocorticotropic hormone (ACTH) stimulation. J Hypertens 2008;26:989-97.

63. Umakoshi H, Naruse M, Wada N, Ichijo T, Kamemura K, Matsuda $\mathrm{Y}$, et al. Adrenal venous sampling in patients with positive screening but negative confirmatory testing for primary aldosteronism. Hypertension 2016;67:1014-9.

64. Wolley MJ, Gordon RD, Ahmed AH, Stowasser M. Does contralateral suppression at adrenal venous sampling predict outcome following unilateral adrenalectomy for primary aldosteronism? A retrospective study. J Clin Endocrinol Metab 2015;100:1477-84.
65. Funder JW, Carey RM, Fardella C, Gomez-Sanchez CE, Mantero F, Stowasser M, et al. Case detection, diagnosis, and treatment of patients with primary aldosteronism: an endocrine society clinical practice guideline. J Clin Endocrinol Metab 2008;93:3266-81.

66. Deinum J, Riksen NP, Lenders JW. Pharmacological treatment of aldosterone excess. Pharmacol Ther 2015;154:120-33.

67. Steichen O, Lorthioir A, Zinzindohoue F, Plouin PF, Amar L. Outcomes of drug-based and surgical treatments for primary aldosteronism. Adv Chronic Kidney Dis 2015;22:196-203.

68. Rossi GP. Diagnosis and treatment of primary aldosteronism. Rev Endocr Metab Disord 2011;12:27-36.

69. Gromotowicz-Poplawska A, Szoka P, Kolodziejczyk P, Kramkowski K, Wojewodzka-Zelezniakowicz M, Chabielska E. New agents modulating the renin-angiotensin-aldosterone system-will there be a new therapeutic option? Exp Biol Med (Maywood) 2016;241:1888-99.

70. Fassnacht M, Arlt W, Bancos I, Dralle H, Newell-Price J, Sahdev A, et al. Management of adrenal incidentalomas: European Society of Endocrinology Clinical Practice Guideline in collaboration with the European Network for the Study of Adrenal Tumors. Eur J Endocrinol 2016;175:G1-34.

71. Celen O, O’Brien MJ, Melby JC, Beazley RM. Factors influencing outcome of surgery for primary aldosteronism. Arch Surg 1996;131:646-50.

72. Sawka AM, Young WF, Thompson GB, Grant CS, Farley DR, Leibson C, et al. Primary aldosteronism: factors associated with normalization of blood pressure after surgery. Ann Intern Med 2001;135:258-61.

73. Letavernier E, Peyrard S, Amar L, Zinzindohoué F, Fiquet B, Plouin PF. Blood pressure outcome of adrenalectomy in patients with primary hyperaldosteronism with or without unilateral adenoma. J Hypertens 2008;26:1816-23.

74. Meyer A, Brabant G, Behrend M. Long-term follow-up after adrenalectomy for primary aldosteronism. World J Surg 2005;29:155-9.

75. Catena C, Colussi GL, Di Fabio A, Sechi LA. Mineralocorticoid antagonists treatment versus surgery in primary aldosteronism. Horm Metab Res 2010;42:440-5.

76. Wang W, Hu W, Zhang X, Wang B, Bin C, Huang H. Predictors of successful outcome after adrenalectomy for primary aldosteronism. Int Surg 2012;97:104-11. 